\title{
A CLASS OF DIFFERENTIAL-FUNCTIONAL SYSTEMS. I( $\left.{ }^{1}\right)$
}

\author{
BY
}

ROBERT B. KELMAN

\section{INTRODUCTION}

1.1. Background. In this series of papers we shall discuss the behavior of the real solutions of a certain class of first order differential-functional systems. As the simplest example of the type of system to be studied we list the differential-functional equation

$$
y^{\prime}(x)=g(x) \cos (u x+v y(p x+q)+w)
$$

where $u, v, w, p$ and $q$ are real constants with $p>1$ or $p \geqq 1$ and $q \geqq 0$, and $g(x)$ is a given function. We use ' to denote differentiation with respect to the indicated argument. More generally the systems to be considered are of the form

$$
y^{\prime}(x)=\Lambda(x, y)
$$

where $\Lambda=\left(\Lambda_{1}, \cdots, \Lambda_{k}\right)$ is a certain function of $x$ and functional of $y=\left(y_{1}, \cdots, y_{k}\right)$. Each $\Lambda_{\kappa}(\kappa=1, \cdots, k)$ consists of a sum of terms each of which is a given function of $x$ and functional of $y$, say $g(x, y)$, multiplied by unity or by a cosine whose argument is a finite linear combination of $x$ and $y_{k}\left(x_{\sigma}\right)(\kappa=1, \cdots, k ; \sigma=0,1, \cdots)$ where $x_{0}=x$ and

$$
x_{\sigma+1}=p x_{\sigma}+q
$$

$$
(\sigma=0,1, \cdots)
$$

and where we assume $p>1$ or $p \geqq 1$ and $q>0$. If all the functions $g(x, y)$ referred to above are independent of $y$ we speak of the case with independency, otherwise the case with dependency. If the argument of the above mentioned cosine does not contain $y_{\kappa}\left(x_{0}\right)(\kappa=1, \cdots, k)$ and if the choice $p=1$ and $q=0$ is excluded, we speak of the case with pure translations, otherwise mixed translations. The precise form of the system is given in $\$ 1.4$. Throughout Part I we limit ourselves to the case with independency and pure translation and confine our attention to the asymptotic behavior of the systems (2).

Although these systems may appear a bit novel at first glance, they represent a natural generalization of a system of two first order differential equations that arises upon a suitable polar coordinate transformation of the differential equation

Received by the editors June 17, 1959 and, in revised form, October 9, 1959. tion.

(1) This work was supported in part by Grant No. G1884 of the National Science Founda- 


$$
y^{\prime \prime}(x)+f(x) y^{\prime}(x)+(1+g(x)) y(x)=0 .
$$

It can be seen $[1 ; 2]$ that under general circumstances the substitutions

$$
y(x)=r(x) \sin (x+\theta(x)), \quad y^{\prime}(x)=r(x) \cos (x+\theta(x))
$$

transform (3) into the system

$$
\theta^{\prime}(x)=\Lambda(x, \theta), \quad \frac{r^{\prime}(x)}{r(x)}=P(x, \theta)
$$

where

$$
\begin{aligned}
& \Lambda(x, \theta)=\frac{1}{2}\left\{g(x)+f(x) \cos \left(2 x+2 \theta(x)-\frac{\pi}{2}\right)-g(x) \cos (2 x+2 \theta(x))\right\}, \\
& P(x, \theta)=\frac{-1}{2}\{f(x)+f(x) \cos (2 x+2 \theta(x))+g(x) \cos (2 x+2 \theta(x)-\pi / 2)\} .
\end{aligned}
$$

The above transformation has reduced the problem of finding the solution of (3) to that of finding the solution of $\theta^{\prime}=\Lambda$, and the differential-functional systems we shall study are seen to be a generalization of this differential equation.

1.2. Some results. In $\S 2$ we establish a matrix identity fundamental to these investigations. Using this identity in $\$ 3.1$ we transform $\Lambda(x, y)$, and in $\$ 3.2$ we examine the asymptotic behavior of the solutions. In general this behavior depends on the algebro-number theoretic properties of $p$. In $\$ 3.3$ we consider some applications. We list here a result obtained for equation (1.1-1) which is a special case of an equation occurring in \$3.3. This result typifies the investigations carried out in this paper.

We suppose that $u \neq 0$ and $v \neq 0$. The second of these suppositions is not significant, but the first is due to the limitations of our method. Assume that the function $g(x)$ appearing in (1.1-1) is a function of bounded variation in $[X, \infty]$ where $X$ is a positive constant and that the total variation of $g(x)$ in $[x, \infty]$ is $O x^{-\delta}$ where $\delta$ is a positive constant and further that $g(x) \rightarrow 0$ as $x \rightarrow \infty$. Then, if $p$ is not the reciprocal of an algebraic integer, we find that each solution $y(x)$ of the given differential-functional equation satisfies an order relation

$$
y(x)=K+O x^{-\delta}
$$

where $K$ is an arbitrary constant.

For each polynomial $C=c_{h} p^{h}+\cdots+c_{1} p+c_{0}$ of degree $h$ with rational integral coefficients define $\Gamma(C)$ to be the sum $\delta \sum_{j=0}^{h}\left|c_{j}\right|$ augmented by $2 \delta$ for each $j, 1 \leqq j \leqq h$, such that $c_{j}=0$. For a given algebraic number $p$ let $\Gamma^{\prime}(p)$ be the infimum of the numbers $\Gamma(C)$ where $C$ runs over all polynomials with rational integral coefficients of which $p$ is a root. Then, if $p$ is the reciprocal of an algebraic integer, each solution of (1.1-1) satisfies an order relation 


$$
y(x)=K+O\left(x^{1-\Gamma^{\prime}(p)}+x^{-\delta}\right) .
$$

Moreover (2) is a "best possible" result in the sense that it does not hold if $\Gamma^{\prime}(p)$ is replaced by a larger number.

1.3. Notational preliminaries. In this section are listed some of the notational conventions we shall employ in this series of papers. Unless otherwise stated, all vectors are real. Let $z=\left(z_{1}, \cdots, z_{h}\right)$ be a vector (finite or infinite). We take $|z|=\sum_{j=1}^{h}\left|z_{j}\right|$ to be the norm of $z$. By $X$ we denote a fixed positive number whose value is chosen in accordance with the problem at hand. We put $\int f=\int_{X}^{x} f(t) d t$ for all functions $f$ integrable on any closed subinterval of $[X, \infty)$. If $y(x)=\left(y_{1}(x), \cdots, y_{h}(x)\right)$ and $z(x)=\left(z_{1}(x), \cdots, z_{h}(x)\right)$, then $y(x)=O z(x)$ means $y_{j}(x)=O z_{j}(x)(j=1, \cdots, h)$. Throughout this investigation $k, l, m$ all greater than or equal to 1 and $n$ greater than or equal to 0 denote fixed integers. Unless otherwise stated, $\kappa, \lambda, \mu$ and $\nu$ denote indices that run over the integers $1, \cdots, k ; 1, \cdots, l ; 1, \cdots, m$; and $1, \cdots, n$ respectively while $\sigma$ denotes an index that runs over the non-negative integers. We denote by $f_{\lambda}(x, y)$ and $g_{\mu}(x, y)$ certain given functions of $x$ and functionals of $y$ with $y$ itself being a function of $x$. Further, $f(x, y)$ and $g(x, y)$ denote respectively the vectors $\left(f_{1}, \cdots, f_{l}\right)$ and $\left(g_{1}, \cdots, g_{m}\right)$, while $F(x, y)$ and $G(x, y)$ denote respectively $\left(f\left(x_{0}, y\right), f\left(x_{1}, y\right), \cdots\right)$ and $\left(g\left(x_{0}, y\right), g\left(x_{1}, y\right), \cdots\right)$. $\mathfrak{P}_{\sigma}$ denotes a semi-group under addition possessing a neutral element 0 . By $\mathfrak{P}^{*}$ we denote the strong direct product and by $\mathfrak{B}$ the weak direct product of the semi-groups $\mathfrak{P}_{\sigma}$. We say that $z=(z(0), z(1), \cdots) \in \mathfrak{B}^{*}$ has length $r$ if $r$ denotes the least integer such that $\sigma>r$ implies $z(\sigma)=0$. If there is no such integer $r$, we put len $z=\infty$. Let $\mathfrak{P}^{+}$and $\mathfrak{P}^{-}$denote realizations of $\mathfrak{P}^{*}$ and let $\left(\mathfrak{z}^{+}, z^{-}\right)$be an element of the Cartesian product of $\mathfrak{B}^{+}$by $\mathfrak{B}^{-}$. Then we put len $\left(z^{+}, z^{-}\right)$equal to the larger of len $z^{+}$and len $z^{-}$. The symbols $\Omega=\{\mathfrak{f}\}$, $\mathfrak{l}=\{\mathfrak{l}\}, \mathfrak{M}=\{\mathfrak{m}\}$, and $\mathfrak{N}=\{\mathfrak{n}\}$ denote the realizations of $\mathfrak{P}$ when each of the semi-groups $\mathfrak{B}_{\sigma}$ is put equal to the real $k$-, $l$-, $m$ - and $n$-dimensional vector space respectively. We employ the convention $\exp \{a ; b\}=b^{a}$. For $\mathfrak{I} \in \mathbb{R}$ and $\mathfrak{m} \in \mathfrak{M}$ we define respectively the scalar product $\mathfrak{l} F(x, y)$ and the exponential function $\exp \{m ; G(x, y)\}$ by

$$
\begin{aligned}
\mathfrak{l} \cdot F(x, y) & =\sum_{\lambda \sigma} \mathfrak{l}_{\lambda}(\sigma) f_{\lambda}\left(x_{\sigma}, y\right), \\
\exp \{\mathfrak{m} ; G(x, y)\} & =\prod_{\mu \sigma} \exp \left\{\mathfrak{m}_{\mu}(\sigma) ; g_{\mu}\left(x_{\sigma}, y\right)\right\} .
\end{aligned}
$$

Finally, in the case that all the $\mathfrak{B}_{\sigma}$ are equal we define a translation operator $E$ of domain $\mathfrak{P}^{*}$ by $E(\mathfrak{z}(0), \mathfrak{z}(1), \cdots)=(0, \mathfrak{z}(0), \mathfrak{z}(1), \cdots)$ for $z \in \mathfrak{P}^{*}$. Observe the following rules of computation

$$
\mathfrak{l} \cdot F\left(x_{h}, y\right)=\left(E^{h \mathfrak{l}}\right) \cdot F(x, y), \exp \left\{\mathfrak{m} ; G\left(x_{h}, y\right)\right\}=\exp \left\{E^{h} \mathfrak{m} ; G(x, y)\right\}
$$

where $h \geqq 0$ denotes an integer. 
1.4. Form of the system. Let $B_{\kappa}$ denote a finite set and put $B=\{\beta\}$ $=U_{k} B_{k}$. If $\beta \in B_{k}$ we say the index of $\beta$ is $\kappa$ and write ind $\beta=\kappa$. To each $\beta$ we associate $\bar{\beta}$ a complex number and $\left(\beta^{\prime}, \beta^{\prime \prime}\right)$ an element of $\mathfrak{R} \times \mathfrak{M}$ with nonnegative integer components and $\left|\beta^{\prime \prime}\right|>0$. We define $\phi(\beta, x, y)$ by

$$
\phi(\beta, x, y)=\exp \left\{\beta^{\prime \prime} ; G(x, y)\right\} \cdot \exp \left(i \beta^{\prime} \cdot F(x, y)\right) .
$$

We of ten shorten $\phi(\beta, x, y)$ to $\phi(\beta)$. Let us define $\Lambda_{\kappa}(x, y)$ as the sum $\sum \bar{\beta} \phi(\beta)$ taken over all $\beta \in B_{\boldsymbol{k}}$. We prescribe that $f_{\lambda}(x, y)$ is a function of $x$ and functional of $y$ of the form $f_{\lambda}(x, y)=u_{\lambda} x+\sum_{\kappa} v_{\lambda_{k}} y_{\kappa}(x)+w_{\lambda}$ where $u_{\lambda} \neq 0, v_{\lambda \kappa}$, and $w_{\lambda}$ are real constants. Further we assume that for $\beta \in B$ with $\beta^{\prime} \neq 0$ one has: if $\sigma_{0}$ is the first integer $\sigma$ such that $\beta^{\prime}(\sigma) \neq 0$, then

$$
\sum_{\kappa}\left|\sum_{\lambda} \beta_{\lambda}^{\prime}\left(\sigma_{0}\right) v_{\lambda_{\alpha}}\right| \neq 0, \quad \sum_{\lambda} \beta_{\lambda}^{\prime}\left(\sigma_{0}\right) u_{\lambda} \neq 0 .
$$

The integer $s$ is defined to be the $\sup \left\{\right.$ len $\left.\left(\beta^{\prime}, \beta^{\prime \prime}\right): \beta \in B\right\}$.

As an illustration consider the following differential-functional system which is examined in $\$ 3.3$.

$$
\begin{aligned}
& y_{1}^{\prime}(x)=g_{1}(x)+g_{2}(x) \cos \left(u_{1} x_{1}+v_{11} y_{1}\left(x_{1}\right)+v_{21} y_{2}\left(x_{1}\right)+w_{1}\right), \\
& y_{2}^{\prime}(x)=g_{3}(x)+g_{4}(x) \cos \left(u_{2} x_{2}+v_{12} y_{1}\left(x_{2}\right)+v_{22} y_{2}\left(x_{2}\right)+w_{2}\right) .
\end{aligned}
$$

Clearly $k=l=2$, while $m=4$. To be able to write the system in the form $(1.1-2)$, we construct the table given below.

$B$ will consist of eight elements ${ }_{1} \beta,{ }_{2} \beta, \cdots,{ }_{8} \beta$.

\begin{tabular}{cccccc} 
& ind & $\bar{\beta}$ & $\beta^{\prime}(1)$ & $\beta^{\prime}(2)$ & $\beta^{\prime \prime}(0)$ \\
\hline- & 1 & $1 / 2$ & $(0,0)$ & $(0,0)$ & $(1,0,0,0)$ \\
${ }_{1} \beta$ & 1 & $1 / 2$ & $(1,0)$ & $(0,0)$ & $(0,1,0,0)$ \\
${ }_{3} \beta$ & 2 & $1 / 2$ & $(0,0)$ & $(0,0)$ & $(0,0,1,0)$ \\
${ }_{5} \beta$ & 2 & $1 / 2$ & $(0,0)$ & $(0,1)$ & $(0,0,0,1)$
\end{tabular}

Thus $s=2$. Here we employ the convention that any component of $\left(\beta^{\prime}, \beta^{\prime \prime}\right)$ not appearing in the table is zero. Since

$$
{ }_{2 n} \beta^{\prime}(\sigma)=-{ }_{2 n-1} \beta^{\prime}(\sigma), \quad{ }_{2 n} \beta^{\prime \prime}(\sigma)={ }_{2 n-1} \beta^{\prime \prime}(\sigma),
$$

it is not necessary to include the $\beta$ with even presubscripts in the table.

1.5. Acknowledgments. This series of papers is derived from my doctoral dissertation written at the University of California with the help of the National Science Foundation under Grant Number G1884. I extend my thanks to Professor J. G. van der Corput. Many of the ideas and methods used here were first announced by him in his lectures (as yet unpublished) on asymptotics given at Berkeley during 1956-1957. In these lectures Professor van der Corput discussed the corresponding problem in differential equations, i.e. systems of the type (1.1-2) with $p=1$ and $q=0$. 


\section{The fundamental identity}

2.1. Statement and proof. $A=\{\alpha\}$ denotes a given set, while $A=\{a\}$ denotes a set whose elements are finite tuples of elements of $A$. If $a=\left({ }_{1} \alpha, \cdots,{ }_{N} \alpha\right)$ and $b=\left({ }_{1} \alpha, \cdots,{ }_{N} \alpha,{ }_{N+1} \alpha, \cdots,{ }_{N+M} \alpha\right)$ (not necessarily elements of $A$ ) and $M \geqq 1$, then we say $b$ is a follower of $a$ and write $b>a$. If $M=1$, we say that $b$ is a successor of $a$ and write $b \gg a$. The relation $b \geqq a$ means that $b$ follows $a$ or $b$ is identical to $a$. We partition $A$ into two disjoint subsets, the boundary of $A, \partial A$, and the interior of $A, A^{\circ}$. If $a \in A$ has a successor not in $A$, then $a \in \partial A$. Otherwise we are free to put $a$ in $\partial A$ or $A^{\circ}$. Let $r \geqq 1$ denote a fixed integer. To each finite tuple $a=\left({ }_{1} \alpha, \cdots,{ }_{N} \alpha\right)$ formed by elements of $A$ ( $a$ not necessarily an element of $A$ ) we associate a complex $r^{2}$ matrix $[a]$ such that $[a]=0$, if $\left({ }_{1} \alpha, \cdots,{ }_{N-1} \alpha\right) \notin A^{\circ}$. We denote by $\phi(a)$ and $H(a)$ complex $r$-vector functions defined over $A$ and $A^{\circ}$ respectively. We accept the usual definition of a matrix product and a matrix operating on a vector. For each pair of tuples

$$
a=\left({ }_{1} \alpha, \cdots,{ }_{N} \alpha\right) \text { and } b=\left({ }_{1} \alpha, \cdots,{ }_{N} \alpha, \cdots,{ }_{N+M} \alpha\right)
$$

with $M \geqq 1$ we define

$$
\frac{b !}{a !}=\left[{ }_{1} \alpha, \cdots,{ }_{N+1} \alpha\right]\left[{ }_{1} \alpha, \cdots,{ }_{N+2} \alpha\right] \cdots\left[{ }_{1} \alpha, \cdots,{ }_{N+M} \alpha\right]
$$

and for each $a$ we put $a ! / a$ ! equal to the identity matrix. For $a \in A^{\circ}$ we assume that

$$
\phi(a)=\sum_{0 a}[b]_{\phi}(b)+H(a)
$$

where $\sum_{0 a}$ denotes summation over all $b \gg a$. A sequence, finite or infinite, of the form $(a, 1 \alpha),\left(a,{ }_{1} \alpha,{ }_{2} \alpha\right), \cdots$ is called a chain and the number of elements in a chain is called its length.

TheOREM. Assume: (i) all chains in $A^{\circ}$ are finite; (ii) $a \in A^{\circ}$ implies that the set

$$
\{\alpha: \alpha \in A ;[a, \alpha] \neq 0\}
$$

is finite. We conclude: $a \in A$ implies that

$$
\phi(a)=\sum_{1 a} \frac{b !}{a !} \phi(b)+\sum_{2 a} \frac{b !}{a !} H(b)
$$

where $\sum_{1 a}$ denotes summation over all $b \geqq a$ such that $b \in \partial A$ while $\sum_{2 a}$ denotes summation over all $b \geqq a$ such that $b \in A^{\circ}$.

REMARK. Condition (i) above is certainly satisfied if there exists a number $K$ such that $\left({ }_{1} \alpha, \cdots,{ }_{N} \alpha\right) \in A^{\circ}$ implies that $N \leqq K$.

Proof. To each $a \in A$ we can associate a chain, possibly empty, of the form 
$\left(a,{ }_{1} \alpha\right),\left(a,{ }_{1} \alpha,{ }_{2} \alpha\right), \cdots$ such that each element of the chain is in $A^{\circ}$ and $\left[a,{ }_{1} \alpha\right] \neq 0,\left[a,{ }_{1} \alpha,{ }_{2} \alpha\right] \neq 0, \cdots$. For each $a \in A^{\circ}$ we take $L(a)$ as the length of the longest such chain, and we put $L(a)=0$ if $a \in \partial A$. Note that for $a \in A$ one has that $L(a)$ is finite. Our proof proceeds by induction. If $L(a)=0$ then either (i) $a \in \partial A$ or (ii) $a \in A^{\circ}$ and for each $b \gg a$ one has that $b \in \partial A$, or $b \in A^{\circ}$ and $[b]=0$. If (i) holds, the theorem is true trivially. If (ii) holds, then the sum in (1) is extended over all $b \gg a$ such that $b \in \partial A$. As an induction hypothesis, we assume that (2) holds for each $d \in A$ such that $L(d) \leqq j$. Assume that $L(a)=j+1$. This implies that $a \in A^{\circ}$ so that (1) can be used. Thus

$$
\begin{aligned}
\phi(a) & =\sum_{0 a}\left\{\sum_{1 b}[b] \frac{c !}{b !} \phi(c)+\sum_{2 b}[b] \frac{c !}{b !} H(c)\right\}+H(a) \\
& =\sum_{1 a} \frac{c !}{a !} \phi(c)+\sum_{2 a} \frac{c !}{a !} H(c) .
\end{aligned}
$$

This completes the induction from $j$ to $j+1$. Q.E.D.

\section{The case with amenable variation}

3.1. Transformation of $\Lambda$. In using the Fundamental Identity on $\Lambda$ we take $A$ as the set $\{(\beta, \sigma): \beta \in B ; \sigma \geqq 0\}$ and for $\alpha=(\beta, \sigma)$ put $\bar{\alpha}=\bar{\beta}, \alpha^{\prime}=E^{\sigma} \beta^{\prime}$, $\alpha^{\prime \prime}=E^{\sigma} \beta^{\prime \prime}$, and ind $\alpha=$ ind $\beta$. We prescribe that $A$ contain only tuples of the form $\left((\beta, 0),{ }_{2} \alpha, \cdots,{ }_{N} \alpha\right)$ and that the set $\{(\beta, 0): \beta \in B\}$ is in $A$. For $a=\left({ }_{1} \alpha, \cdots,{ }_{N} \alpha\right)$ we put $a^{\prime}={ }_{1} \alpha^{\prime}+\cdots+{ }_{N} \alpha^{\prime}$ and $a^{\prime \prime}={ }_{1} \alpha^{\prime \prime}+\cdots+{ }_{N} \alpha^{\prime \prime}$ and ind $a=$ ind ${ }_{1} \alpha$. By $A_{\kappa}$ we denote the set $\{a: a \in A$; ind $a=\kappa\}$. We put $\partial A_{\kappa}$ $=A_{\kappa} \cap \partial A$ and $A_{\kappa}^{\circ}=A_{\kappa} \cap A^{\circ}$. The question of the specific construction of $A$ and the satisfaction of the hypothesis of the Fundamental Identity Theorem is discussed in $\$ 3.2$. For the present we assume this hypothesis is fulfilled. For $a \in A$ let us put $a^{\prime} \cdot u=\sum_{\lambda_{\sigma}} a_{\lambda}^{\prime}(\sigma) u_{\lambda} p^{\sigma}$. If $a^{\prime} \cdot u \neq 0$ we define $[a,(\beta, \sigma)]$ by

$$
[a,(\beta, \sigma)]=-\left(a^{\prime} \cdot u\right)^{-1} \bar{\beta} p^{\sigma} \sum_{\lambda} a_{\lambda}^{\prime}(\sigma) v_{\lambda_{\kappa}}
$$

where $\kappa=$ ind $\beta$, and define $H(a)$ by

$$
\begin{aligned}
H(a)= & -\left(a^{\prime} \cdot u\right)^{-1} \phi(a) \sum_{\lambda \kappa \sigma} a_{\lambda}^{\prime}(\sigma) v_{\lambda \kappa} p^{\sigma}\left\{y_{\kappa}^{\prime}\left(x_{\sigma}\right)-\Lambda_{\kappa}\left(x_{\sigma}, y\right)\right\} \\
& -i\left(a^{\prime} \cdot u\right)^{-1} \exp \left\{a^{\prime \prime} ; G(x)\right\} \cdot \frac{d}{d x}\left(\exp i a^{\prime} \cdot F(x, y)\right) .
\end{aligned}
$$

If $a^{\prime} \cdot u \neq 0$, then for any differentiable function $y$ one has

$$
\phi(a)=-\left(a^{\prime} \cdot u\right)^{-1} \phi(a) \sum_{\lambda_{\kappa} \sigma} a_{\lambda}^{\prime}(\sigma) v_{\lambda_{\kappa}} p^{\sigma} \Lambda_{\kappa}\left(x_{\sigma}, y\right)+H(a)
$$

which in terms of the preceding definitions is equivalent to $(2.1-1)$. For $a=\left({ }_{1} \alpha, \cdots,{ }_{N} \alpha\right)$ we put $a !={ }_{1} \bar{\alpha}\left(a ! /{ }_{1} \alpha !\right)$ and call $a$ significant if $a ! \neq 0$. From the Fundamental Identity one obtains 


$$
\Lambda_{\kappa}(x, y)=\sum_{a \in \partial A_{\kappa}} a ! \phi(a)+\sum_{a \in A_{\kappa}} a ! H(a) .
$$

For convenience in the applications we introduce $D_{\kappa}^{\prime \prime}$ a subset of $\mathfrak{M}$. We assume $\mathrm{U}_{\kappa} D_{\kappa}^{\prime \prime}$ contains all points $a^{\prime \prime}$ corresponding to those $a$ such that at least one successor of $a$ is not in $A$. We now make precise our definition of $A_{\kappa}^{\circ}$ :

$$
A_{\kappa}^{\circ}=\left\{a: a \in A_{\kappa} ; a^{\prime} \cdot u \neq 0 ; a^{\prime \prime} \in D_{\kappa}^{\prime \prime}\right\} .
$$

Let $\partial A_{\kappa}$ be partitioned into the following three disjoint sets:

$$
\begin{aligned}
& \left\{a: a \in \partial A_{\kappa} ; a^{\prime \prime} \notin D_{\kappa}^{\prime \prime} ; a^{\prime} \cdot u=0 ; \sum_{\kappa^{\prime} \sigma}\left|\sum_{\lambda} a_{\lambda}^{\prime}(\sigma) v_{\lambda_{\kappa^{\prime}}}\right|=0\right\}, \\
& \left\{a: a \in \partial A_{\kappa} ; a^{\prime \prime} \notin D_{\kappa}^{\prime \prime} ; a^{\prime} \cdot u=0 ; \sum_{\kappa^{\prime} \sigma}\left|\sum_{\lambda} a_{\lambda}^{\prime}(\sigma) v_{\lambda \kappa^{\prime}}\right| \neq 0\right\}, \\
& \left\{a: a \in \partial A_{\kappa} ; a^{\prime \prime} \in D_{\kappa}^{\prime \prime}\right\} .
\end{aligned}
$$

We take $P_{\kappa}^{\prime}(x), Q_{\kappa}^{\prime}(x, y)$, and $R_{\kappa}^{\prime}(x, y)$ as the sum $\sum a ! \phi(a)$, taken respectively, over the elements $a$ given by (1.1), (1.2), and (1.3). Further, $S_{\kappa}^{\prime}(x, y)$ and $T_{\kappa}^{\prime}(x, y)$ are given by

$$
\begin{aligned}
& S_{\kappa}^{\prime}(x, y)=-i \sum a !\left(a^{\prime} \cdot u\right)^{-1} \exp \left\{a^{\prime \prime} ; G(x)\right\} \frac{d}{d x}\left(i a^{\prime} \cdot F(x, y)\right), \\
& T_{\kappa}^{\prime}(x, y)=-\sum a !\left(a^{\prime} \cdot u\right)^{-1} \phi(a) \sum_{\lambda_{\kappa^{\prime} \sigma}} v_{\alpha^{\prime}} p^{\sigma}\left\{y_{\kappa^{\prime}}^{\prime}\left(x_{\sigma}\right)-\Lambda_{\kappa^{\prime}}\left(x_{\sigma}, y\right)\right\}
\end{aligned}
$$

the sums in both cases being extended over all $a \in A_{\kappa}^{\circ}$. Thus each solution $y(x)$ of $y^{\prime}=\Lambda$ satisfies the equation

$$
y(x)=K+P(x)+Q(x, y)+R(x, y)+S(x, y)
$$

where $K$ is a real constant and where $P=\int P^{\prime}$, etc.

DEFINITION. $\Lambda_{\kappa}, 1 \leqq \kappa \leqq k$, satisfies the condition of amenable variation relative to a suitably chosen set $A$ with upper bound $Z_{\kappa}(x)$ if for each significant $a \in A_{\kappa}^{\circ}$ one has that $\exp \left\{a^{\prime \prime} ; G(x)\right\}$ is a function of bounded variation in $[X, \infty]$ such that the total variation of $\exp \left\{a^{\prime \prime} ; G(x)\right\}$ in $[x, \infty]$ is $O Z_{\mathbf{x}}(x)$, and $\exp \left\{a^{\prime \prime}, G(x)\right\} \rightarrow 0$ as $x \rightarrow \infty$. Further, $\Lambda_{\kappa}$ satisfies the condition of amenable integrability relative to a suitably chosen set $A$ with upper bound $Z_{\mathrm{x}}(x)$ if for each significant a occurring in the set $(1.3) \exp \left\{a^{\prime \prime} ; G(x)\right\}$ is absolutely integrable to infinity in such a way that

$$
\int_{\infty}^{x}\left|\exp \left\{a^{\prime \prime} ; G(t)\right\}\right| d t=O Z_{\kappa}(x) .
$$

These definitions plus an appeal to Chartier's Test [3, p. 72] yield the following 
Lemma. If $\Lambda_{\kappa}, 1 \leqq \kappa \leqq k$, satisfies the conditions of amenable variation and integrability with respect to a suitably chosen set $A$ and common upper bound $Z_{k}(x)$, then for each solution $y$ of (1.1-2) $y_{\mathrm{x}}$ satisfies an order relation

$$
y_{\kappa}(x)=K_{\kappa}+P_{\kappa}(x)+Q_{\kappa}(x, y)+O Z_{\kappa}(x) .
$$

If $\Lambda_{\kappa}$ satisfies the condition of amenable variation for each $\kappa, \kappa=1,2, \cdots, k$, with upper bound $Z_{k}$, we say merely that $\Lambda$ satisfies this condition with upper bound $Z=\left(Z_{1}, \cdots, Z_{k}\right)$ and similarly for amenable integrability.

3.2. On the sets $A$ and $D_{\kappa}^{\prime \prime}$. The constructions given in this section are the ones chiefly used in applications. Let $\epsilon_{\kappa}$ and $\delta_{\mu}$ denote positive constants. We define $D_{\mathfrak{k}}^{\prime \prime}$ as the set of all points $\mathfrak{m}$ in $\mathfrak{M}$ such that

$$
\sum_{\mu \sigma} \mathfrak{m}_{\mu}(\sigma) \delta_{\mu} \geqq 1+\epsilon_{\kappa}, \quad \mathrm{m}_{\mu}(\sigma) \geqq 0 .
$$

Put

$$
d=\inf \left\{\sum_{\mu \sigma} \beta_{\mu}^{\prime \prime}(\sigma) \delta_{\mu}: \beta \in B\right\} .
$$

If $a=\left({ }_{1} \alpha, \cdots,{ }_{N} \alpha\right)$ is a significant element of $A_{\kappa}$, certainly $N \leqq d^{-1}\left(1+\epsilon_{\kappa}\right)+1$. We say that the tuple $a=\left({ }_{1} \alpha, \cdots,{ }_{N} \alpha\right)$ has property $\mathrm{I}$ if there exists an integer $j, 2 \leqq j \leqq N$, such that ${ }_{j} \alpha=(\beta, \sigma)$ and

$$
\sum_{\lambda}\left({ }_{1} \alpha_{\lambda}{ }^{\prime}(\sigma)+{ }_{2} \alpha_{\lambda}{ }^{\prime}(\sigma)+\cdots+{ }_{j-1} \alpha_{\lambda}{ }^{\prime}(\sigma)\right) v_{\lambda_{\kappa}}=0
$$

where $\kappa=$ ind ${ }_{j} \alpha$. We define $D_{\kappa}$ as the set of points $a \in A_{\kappa}$ such that $a^{\prime \prime} \in D_{\kappa}^{\prime \prime}$ or $a$ has the property $\mathrm{I}$, and we put $D=\mathrm{U}_{\kappa} D_{\kappa}$. If $a \in A^{\circ}$ and $[a,(\beta, \sigma)] \neq 0$, then $0 \leqq \sigma \leqq$ len $a^{\prime}$. This means there are at most a finite number of elements $(\beta, \sigma)$ such that $[a,(\beta, \sigma)] \neq 0$. Thus using the sets $A$ and $D$ given above the hypothesis of the Fundamental Identity theorem is satisfied. Throughout the remainder of this paper we use the construction for $A$ given above. To determine the nature of $P$ and $Q$ we need the following

Lemma. For each $a \in A_{\kappa}-D_{\kappa}$ the three following assertions are equivalent:
(i) $a^{\prime} \neq 0$;
(ii) $\sum_{\kappa \sigma}\left|\sum_{\lambda} a_{\lambda}^{\prime}(\sigma) v_{\lambda \kappa}\right| \neq 0$;
(iii) $\sum_{\sigma}\left|\sum_{\lambda} a_{\lambda}^{\prime}(\sigma) u_{\lambda}\right| \neq 0$.

Proof. Assume $a=\left({ }_{1} \alpha, \cdots,{ }_{N} \alpha\right)$ denotes an element of $A_{\kappa}-D_{\kappa}$ for which $a^{\prime} \neq 0$. If $N=1$ condition (1.4-1) implies the lemma. Hence, we can assume in the proof that $N \geqq 2$. Since ${ }_{1} \alpha$ is of the form $\left({ }^{1} \beta, 0\right)$, one has that ${ }^{1} \beta^{\prime}=0$ and $N \geqq 2$ imply $a$ has property I. Thus we can also assume without loss of generality that ${ }^{1} \beta^{\prime} \neq 0$. Let $\sigma_{0}$ be the least integer $\sigma$ such that ${ }^{1} \beta^{\prime}(\sigma) \neq 0$. Since $a$ does not have property $\mathrm{I}$, each ${ }_{j} \alpha(j=2,3, \cdots, N)$ is of the form $\left.{ }^{i} \beta, \sigma_{j}\right)$ where $\sigma_{j} \geqq \sigma_{0}$. This implies that the component $\sigma=\sigma_{0}$ of $a^{\prime}$ is just ${ }^{1} \beta^{\prime}\left(\sigma_{0}\right)$. This in conjunction with (1.4-1) proves the lemma. 
Using this lemma we establish the following:

TheOREM. $P_{\star}^{\prime}=\sum \bar{\beta} \phi(\beta)$ where the sum is extended over all $\beta \in B_{\kappa}$ such that $\beta^{\prime}=0$.

Proof. By the preceding lemma $P_{\kappa}^{\prime}=\sum a ! \phi(a)$ where the sum is extended over all $a \in A_{\kappa}-D_{\kappa}$ such that $a^{\prime}=0$. Assume that $a=\left({ }_{1} \alpha, \cdots,{ }_{N} \alpha\right)$ is a significant element of this sum with $N \geqq 2$. Then, ${ }_{1} \alpha$ is of the form $\left({ }^{1} \beta, 0\right)$ where ${ }^{1} \beta^{\prime} \neq 0$. Let $\sigma_{0}$ denote the least integer $\sigma$ such that ${ }^{1} \beta^{\prime}(\sigma) \neq 0$. As before $a^{\prime}\left(\sigma_{0}\right)$ $={ }^{1} \beta^{\prime}\left(\sigma_{0}\right)$. This yields the theorem.

By virtue of the preceding lemma, the equations $a^{\prime} \cdot u=0\left(a \in A_{k}-D_{k} ; a^{\prime} \neq 0\right)$ possess at most $a$ finite roots. If we choose $p$ so that it is not one of those roots, then $Q_{k}=0$. In particular if $p$ is not a member of the algebraic closure of the extension of the rationals formed by adjoining $u_{1}, \cdots, u_{l}$, then $Q=0$. Thus we obtain the following

Corollary. Assume: (i) $\beta^{\prime} \neq 0(\beta \in B)$; (ii) the conditions of amenable variation and integrability hold with common upper bound $Z(x)=o(1)$; (iii) $p$ is not an element of the algebraic closure of the extension of the rationals formed by adjoining $u_{1}, \cdots, u_{l}$. Then each solution $y$ of $y^{\prime}=\Lambda$ tends to a finite limit as $x \rightarrow \infty$. More precisely it satisfies an order relation $y=K+O Z$.

In passing let us note that if the numbers $u_{1}, \cdots, u_{l}$ are algebraic integers (or $u_{1}=u_{2}=\cdots=u_{l}$ ) and if

$$
\sum_{\lambda} \beta_{\lambda}^{\prime}\left(\sigma_{0}\right) u_{\lambda}=\text { algebraic unit } \quad\left(\beta \in B_{\kappa} ; \beta^{\prime} \neq 0\right)
$$

where $\sigma_{0}$ denotes the least integer $\sigma$ such that $\beta^{\prime}(\sigma) \neq 0$, then if $p$ is not the reciprocal of an algebraic integer, $Q_{\kappa}=0$.

Let us now consider for given $\kappa, 1 \leqq \kappa \leqq k$, the possibility that $p$ is the root of an equation

$$
c_{h} p^{h}+c_{h-1} p^{h-1}+\cdots+c_{1} p+c_{0}=0
$$

with the following property: there exists at least one element

$$
a=\left({ }_{1} \alpha, \cdots,{ }_{N} \alpha\right) \text { of } A_{\kappa}-D_{\kappa} \text { with }{ }_{1} \alpha=\left({ }^{1} \beta, 0\right)
$$

such that $a^{\prime} \neq 0$ and if $\sigma_{0}$ denotes the least integer $\sigma$ such that ${ }^{1} \beta^{\prime}(\sigma) \neq 0$ then

$$
\begin{aligned}
c_{j} & =\sum_{\lambda} a_{\lambda}^{\prime}\left(\sigma_{0}+j\right) u_{\lambda} \quad(j=0,1, \cdots, h), \\
a^{\prime}(\sigma) & =0 \quad\left(\sigma \geqq \sigma_{0}+h+1\right) .
\end{aligned}
$$

Let $E_{\kappa}$ denote the totality of elements $a$ of $A_{\kappa}-D_{\kappa}$ with the above mentioned property. Then there exists an element $b_{\kappa}$ of $E_{\kappa}$ such that $\exp \left\{a^{\prime \prime} ; G(x)\right\}$ $=O \exp \left\{b_{\kappa}^{\prime \prime} ; G(x)\right\}\left(a \in E_{\mathrm{x}}\right)$. In this case $Q_{\kappa}^{\prime}=O \exp \left\{b_{\kappa}^{\prime \prime} ; G(x)\right\}$. Theoretically one could always determine such an element $b_{\kappa}$ by examination of all the possible elements $a$ of $A_{\kappa}-D_{\kappa}$ once the number $p$ had been given. How- 
ever, if the algebraic order of $p$ is large, or if $k, l$, or $m$ is large, the task is nontrivial. In a future work we shall consider the computational aspects of this problem.

3.3. Applications. Consider the equation

$$
y^{\prime}(x)=g_{l+1}(x)+\sum_{\lambda=1}^{l} g_{\lambda}(x) \cos \left(u_{\lambda} x_{1}+v_{\lambda_{1}} y\left(x_{1}\right)+w_{1}\right) .
$$

Here $g_{\lambda}(x)(\lambda=1,2, \cdots, l+1)$ is a function of bounded variation in $[X, \infty]$ such that the total variation of $g_{\lambda}(x)$ in $[x, \infty]$ is $O \exp \left\{-\delta_{\lambda} ; x\right\}$, where $\delta_{\lambda}$ is a positive constant, and such that $g_{\lambda}(x) \rightarrow 0$ as $x \rightarrow \infty$. We observe that $k=1, m=l+1$ and $s=1$. Also $B$ consists of $2 m$ elements ${ }_{j} \beta(j=1,2, \cdots, 2 m)$. The table for the equation is

$$
\begin{gathered}
{ }_{2 j-1} \bar{\beta}=\frac{1}{2}, \quad{ }_{2 j-1} \beta_{j}^{\prime}(1)=1, \quad{ }_{2 j-1} \beta_{j}^{\prime \prime}(0)=1, \quad(j=1, \cdots, m) \\
2 l+1 \bar{\beta}=\frac{1}{2}, \quad{ }_{2 l+1} \beta^{\prime \prime}(0)=1 .
\end{gathered}
$$

We denote by $\delta$ the infimum of the numbers $\delta_{1}, \delta_{2}, \cdots, \delta_{l}$. Let us put $\epsilon_{1}$ of $\S 3.2$ equal to $\delta$. The numbers $\delta_{\lambda}(\lambda=1, \cdots, l+1)$ in this section coincide with numbers $\delta_{\mu}(\mu=1, \cdots, m)$ of $\S 3.2$. We have at once that the conditions of amenable variation and integrability hold with upper bound $\exp \{-\delta ; x\}$, and that $P_{1}=\int g_{l+1}$. Let $r_{i j}(i, j=1, \cdots, l)$ denote the ratio $u_{i} / u_{j}$. Consider a polynomial $C$ of the form

$$
\left(\sum_{i, j=1}^{l} c_{i j}^{h} r_{i j}\right) p^{h}+\cdots+\left(\sum_{i, j=1}^{l} c_{i j}^{1} r_{i j}\right) p+1
$$

where the numbers $c_{i j}^{t}(i, j=1, \cdots, l ; t=1, \cdots, h)$ run over the rational integers subject to the restriction that if $c_{i j^{\prime}}^{i} \neq 0$ for some $j^{\prime}$, then $c_{i}^{i}=0$ for all $j \neq j^{\prime}$ and where $h$ denotes an arbitrary positive integer. We employ the convention that if $j^{\prime}$ is the number referred to just above, then $c^{0}{ }^{\prime} j^{\prime}=1$ and $c_{i j}^{0}=0$ if $i \neq j^{\prime}$ or $j \neq j^{\prime}$. In expressing a polynomial in the form (2) the choice of the coefficients $c_{i j}^{i}$ is not unique. It is convenient for our purpose to regard two polynomials, say $C_{1}$ and $C_{2}$, as distinct if not all the coefficients $c_{i j}^{i}$ used in expressing the two polynomials are identical even though $C_{1}$ and $C_{2}$ may be equal in the ordinary sense. To continue this investigation we must first show a side result: if $a \in A_{1}-D_{1}$ and len $a^{\prime}=N \geqq 1$, then $d=\sum_{\lambda=1}^{l} \sum_{\sigma} a_{\lambda}^{\prime \prime}(\sigma)$ is greater than or equal to $\left|a^{\prime}\right|$ augmented by 2 for each integer $\sigma, 1 \leqq \sigma$ $\leqq N-1$, such that $a^{\prime}(\sigma)=0$. It is clear that $d \geqq\left|a^{\prime}\right|$. Assume that $a=\left({ }_{1} \alpha, \cdots,{ }_{M} \alpha\right)$. There is an integer $j(0), 1 \leqq j(0) \leqq M$, such that ${ }_{j(0)} \alpha$ is of the form $(\beta, N-1)$ where $\beta^{\prime} \neq 0$. In order that $a$ does not have property I, there exists for each integer $\tau, 2 \leqq \tau \leqq N$, an integer $j(\tau), 1 \leqq j(\tau) \leqq M$, such that $j(\tau) \alpha$ is of the form $(\beta, N-\tau)$ where $\beta^{\prime} \neq 0$. Thus corresponding to each $\sigma$, 
$1 \leqq \sigma \leqq N-1$ such that $a^{\prime}(\sigma)=0$ there are at least two distinct elements $\alpha$ of the tuple $a$ both of the form $(\beta, \sigma-1)$ where $\beta^{\prime} \neq 0$. This yields the above mentioned result.

For each distinct polynomial $C$ of the form (2) and each $p$ we introduce a function $\Gamma(C, p)$ defined as follows: $\Gamma(C, p)$ equals the sum $\sum_{i=0}^{h} \sum_{i, j=1}^{l}\left|c_{i j}^{t}\right| \delta_{i}$ augmented by $2 \delta$ for each integer $t, 1 \leqq t \leqq h$, such that $\sum_{t, j=1}^{l}\left|c_{i, j}^{t}\right|=0$ provided that (i) $C(p)=0$ and (ii) there exists $a \in A_{1}-D_{1}$ such that for the above mentioned integer $j^{\prime}$ one has that

$$
\begin{aligned}
a^{\prime}(\sigma+1) & =\left(\underset{c_{1 j^{\prime}}}{\sigma}, \underset{c_{2 j^{\prime}}}{\sigma}, \cdots, \underset{c_{l j^{\prime}}}{\sigma}, 0\right) & (\sigma=0,1, \cdots, h), \\
a^{\prime}(\sigma) & =0 & (\sigma \geqq h+2) ;
\end{aligned}
$$

if there exists no such element $a$ or if $C(p) \neq 0$, we put $\Gamma(C, p)=\infty$. For a given $p$ let us put $\Gamma^{\prime}(p)$ equal to the infimum of the numbers $\Gamma(C, p)$ where $C$ runs over all distinct polynomials of the form (2). Then each solution $y$ satisfies an order relation

$$
y(x)=K+\int g_{l+1}+O\left(x^{-\delta}+x^{1-\Gamma^{\prime}(p)}\right)
$$

where $\exp \left\{1-\Gamma^{\prime}(p) ; x\right\}$ is replaced by $\log x$ if $1=\Gamma^{\prime}(p)$. For each positive $\delta$ one has $\Gamma^{\prime}(p)=\infty$ except for a finite number of $p$. In the particular case $u_{1}=u_{2}=\cdots=u_{l}$ we see by $(3.2-2)$ that if $p$ is not the reciprocal of an algebraic integer then (2) is valid with $\Gamma^{\prime}(p)=\infty$ for all positive $\delta$. For the special case of equation (1.1-1) this yields (1.2-1) and (1.2-2). We can improve the result for (1.1-1) in the case that $p=1$ and $q>0$. For the elements $a_{1}=\left(\left({ }_{1} \beta, 0\right),\left({ }_{2} \beta, 1\right)\right)$ and $a_{2}=\left(\left({ }_{2} \beta, 0\right),\left({ }_{1} \beta, 1\right)\right)$ one has $a_{1} !=a_{2} !=-v / 4 u$. By the mean value theorem

$$
y(x+2 q)-y(x+q)=O x^{-\delta} .
$$

Hence, $Q_{1}$ is given by

$$
-\frac{v}{2 u} \cos (u q) \int_{X}^{x} g\left(t_{1}\right) g(t) d t+O x^{1-3 \delta}
$$

where $\exp \{1-3 \delta ; x\}$ is replaced by $\log x$ if $\delta=1 / 3$. Thus each real solution $y(x)$ satisfies an order relation

$$
y(x)=K-\frac{v}{2 u} \cos (u q) \int_{X}^{x} g\left(t_{1}\right) g(t) d t+O\left(x^{-\delta}+x^{1-3 \delta}\right),
$$

again $\exp \{1-3 \delta ; x\}$ being replaced by $\log x$ if $\delta=1 / 3$. This shows that in general $\Gamma^{\prime}(p)$ cannot be replaced by a larger number so that (3) represents a "best possible" result.

As a second example we consider the system (1.4-2) and assume $g_{\mu}(x)(\mu=1,2,3,4)$ is a function of bounded variation in $[X, \infty]$ such that 
$g_{\mu}(x) \rightarrow 0$ as $x \rightarrow \infty$ and the total variation of $g_{\mu}(x)$ in $[x, \infty]$ is $O\left(\exp \left\{-\delta_{\mu} ; x\right\}\right)$ where $\delta_{\mu}$ is a positive constant. Again using the notation of $\$ 3.2$ we put $\epsilon_{1}=\delta_{2}$ and $\epsilon_{2}=\delta_{4}$. Consider a polynomial $C$ of the form

$$
\left(c_{1 h}+c_{2 h} \frac{u_{2}}{u_{1}}\right) p^{h}+\cdots+\left(c_{11}+c_{21} \frac{u_{2}}{u_{1}}\right) p+1
$$

where the numbers $c_{i j}$ denote arbitrary integers.

$C$ is said to have an $\alpha$-gap of length $t$ if there is a positive integer $i$ such that

$$
\begin{aligned}
& \left|c_{1, i+\nu}\right|+\left|c_{2, i+\nu}\right|=0 \quad(\nu=0,1, \cdots, t-1), \\
& \left|c_{1, i-1}\right|+\left|c_{2, i-1}\right| \neq 0 ; \quad c_{1, i+t}=0, \quad c_{2, i+t} \neq 0 .
\end{aligned}
$$

The gap is called a $\beta$-gap if the last two relations in (6) are replaced by $c_{1, i+t} \neq 0, c_{2, i+t}=0$ and an $\alpha \beta$-gap if these relations are replaced by $c_{1, i+t} \neq 0$, $c_{2, i+i} \neq 0$. A gap is called anticipated if $\left|c_{1, i-2}\right|+\left|c_{2, i-2}\right|=0$ where $i-2$ is positive and unanticipated otherwise. We recall the agreement on distinct polynomials used in the preceding" example. For each distinct polynomial $C$ and each $p$ we define a function $\Gamma(C, p)$ : if (i) $C(p) \neq 0$ or (ii) there exists no $a \in A_{1}-D_{1}$ such that

$$
a^{\prime}=\left(0,( \pm 1,0),\left(c_{11}, c_{21}\right),\left(c_{12}, c_{22}\right), \cdots,\left(c_{1 h}, c_{2 h}\right), 0,0, \cdots\right)
$$

then we put $\Gamma(C, p)=\infty$; if (i) $C(p)=0$ and (ii) if there is $a \in A_{1}-D_{1}$ with (5) then we put $\Gamma(C, p)$ equal to the sum

$$
\sum_{t=0}^{h}\left|c_{1 t}\right| \delta_{2}+\left|c_{2 t}\right| \delta_{4}
$$

augmented as follows: for each anticipated $\alpha$-gap in $C$ of length $2 t$ and anticipated $\beta$-gap of length $2 t-1$ increase the sum (6) by

$$
\inf \left\{2(2 t-1-2 j) \delta_{2}+2 j \delta_{4}: j=0,1, \cdots, t-1\right\} ;
$$

for each anticipated and unanticipated $\alpha$-gap of length $2 t+1$, for each anticipated and unanticipated $\beta$-gap of length $2 t$ increase the sum (6) by

$$
\inf \left\{4(t-j) \delta_{2}+2 j \delta_{4}: j=0,1, \cdots, t\right\} ;
$$

for each unanticipated $\alpha$-gap of length $2 t$ and for each unanticipated $\beta$-gap of length $2 t-1$ increase the sum (6) by

$$
\inf \left\{2(2 t-1-2 j) \delta_{2}+2 j \delta_{4}: j=0,1, \cdots, t-1 ; 2 t \delta_{4}\right\} ;
$$

for each $\alpha \beta$-gap increase the sum (6) by the lesser of the corresponding amounts given above for $\alpha$ - and $\beta$-gaps. For each choice of $p$ denote by $\Gamma^{\prime}(p)$ the infimum of the numbers $\Gamma(C, p)$ where $C$ runs over the polynomials of the given type. We find that for each solution $y$, one has 


$$
y_{1}(x)=K_{1}+\int g_{1}+O\left(x^{1-\Gamma^{\prime}(p)}+x^{-\delta_{2}}\right)
$$

where $\exp \left\{1-\Gamma^{\prime}(p) ; x\right\}$ is replaced by $\log x$ if $1=\Gamma^{\prime}(p)$. A similar result can be obtained for $\kappa=2$. Denote by $\left({ }^{*}\right)$ the relation $(7)$ with $\Gamma^{\prime}(p)=\infty$. Then, if $u_{2} / u_{1}$, is an algebraic integer, $\left(^{*}\right)$ holds, if $p$ is not the reciprocal of an algebraic integer, for all positive $\delta_{\mu}$. If $u_{2} / u_{1}$ is not an algebraic integer, then the set of $p$ for which we can assert that $\left(^{*}\right)$ holds for all positive $\delta_{\mu}$ is a proper subset of the reciprocals of the algebraic integers. Since similar remarks hold for $\kappa=2$, we find that the strongest results, in the sense that a relation of the type $\left({ }^{*}\right)$ holds for $\kappa=1$ and $\kappa=2$ for all positive $\delta_{\mu}$ with fewest $p$ excluded, occurs in the case that $u_{2} / u_{1}$ is an algebraic unit.

\section{REFERENCES}

1. J. G. van der Corput, Asymptotic expansions III-The asymptotic behavior of certain second order differential equations, Air Force Contract AF-18(600)-958 (R-354-10-52), University of California, 1955.

2. Heinz Prüfer, Neue Herleitung der Sturm-Ljouvilleschen Reihentwicklung stetiger Funktionen, Math. Ann. vol. 95 (1926) pp. 499-518.

3. E. T. Whittaker and G. N. Watson, Modern analysis, 4th ed., Cambridge University Press, 1952.

InTERnational Business Machines Corporation,

YorkTOWN HeIGHTS, NEW YORK 